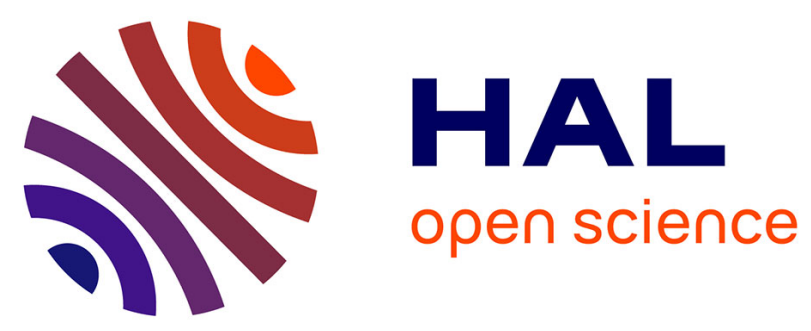

\title{
Influence of strain conditions on the grain sub-structuration in crept uranium dioxide pellets
}

M. Ben Saada, X. Iltis, N. Gey, B. Beausir, A. Miard, P. Garcia, N. Maloufi

\section{To cite this version:}

M. Ben Saada, X. Iltis, N. Gey, B. Beausir, A. Miard, et al.. Influence of strain conditions on the grain sub-structuration in crept uranium dioxide pellets. Journal of Nuclear Materials, 2019, 518, pp.265-273. 10.1016/j.jnucmat.2019.02.052 . cea-02339999

\section{HAL Id: cea-02339999 https://hal-cea.archives-ouvertes.fr/cea-02339999}

Submitted on 9 Dec 2019

HAL is a multi-disciplinary open access archive for the deposit and dissemination of scientific research documents, whether they are published or not. The documents may come from teaching and research institutions in France or abroad, or from public or private research centers.
L'archive ouverte pluridisciplinaire HAL, est destinée au dépôt et à la diffusion de documents scientifiques de niveau recherche, publiés ou non, émanant des établissements d'enseignement et de recherche français ou étrangers, des laboratoires publics ou privés. 


\title{
Influence of strain conditions on the grain sub-structuration in crept uranium dioxide pellets
}

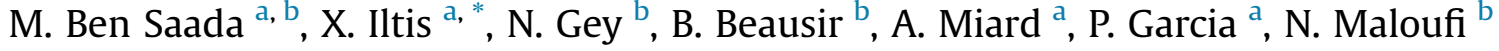 \\ ${ }^{a}$ CEA, DEN, DEC, Cadarache, 13108, Saint-Paul-Lez-Durance, France \\ b Laboratoire D’Etude des Microstructures et de Mécanique des Matériaux (LEM3), UMR CNRS 7239, Université de Lorraine, 57045, Metz Cedex 1, France
}

\section{H I G H L I G H T S}

- Dislocational creep in $\mathrm{UO}_{2}$ leads to the occurrence of sub-boundaries inside the prior grains.

- These sub-boundaries are detected by EBSD, to disorientations as low as $0.25^{\circ}$, and their linear fraction is quantified.

- This fraction increases significantly with the strain rate and strain level.

- $\mathrm{UO}_{2}$ is subject to a dynamic recovery mechanism by progressive increase of sub-boundaries disorientation angle.

\begin{abstract}
A B S T R A C T
Stoichiometric uranium dioxide $\left(\mathrm{UO}_{2}\right)$ pellets were deformed by uniaxial compression creep tests at $1500{ }^{\circ} \mathrm{C}$. Strains comprised between $3 \%$ and $11 \%$ were applied at strain rates ranging from $\sim 5 \times 10^{-6} \mathrm{~s}^{-1}$ to $70 \times 10^{-6} \mathrm{~s}^{-1}$ to deform pellets in the dislocational creep regime. An optimized protocol for Electron BackScattered Diffraction (EBSD) data acquisition and processing was applied to detect and quantify low and very low angle sub-boundaries disoriented down to $0.25^{\circ}$ inside the prior $\mathrm{UO}_{2}$ grains. Three EBSD based parameters were calculated and are efficient to quantify the evolution of the deformed microstructure with the deformation conditions: the linear fraction of the sub-boundaries, the corresponding Geometrically Necessary Dislocations (GNDs) density and the resulting mean sub-grain size. The linear fraction of sub-boundaries (and their transcription in GNDs) increased significantly with the strain level and rate. New very low angle boundaries disoriented by less than $1^{\circ}$ were continuously created whereas already existing sub-boundaries increased continuously their disorientation. This microstructural evolution can be described as a dynamic recovery mechanism by progressive increase of sub-boundaries disorientation angle.
\end{abstract}

\section{Introduction}

In nuclear Pressurized Water Reactors (PWRs), during power transients, sintered uranium dioxide $\left(\mathrm{UO}_{2}\right)$ pellets undergo viscoplastic deformation by creep mechanisms, which influence pelletcladding interaction processes [1,2]. During transients, the temperature reaches more than $1500^{\circ} \mathrm{C}$ in the central part of the pellets, allowing the occurrence of creep mechanisms. These mechanisms can be at least partly reproduced by means of compression creep tests on non-irradiated pellets. Different conditions of temperature, strain rate and stress are reported in the

\footnotetext{
* Corresponding author.

E-mail address: xaviere.iltis@cea.fr (X. Iltis).
}

literature with resulting data usually expressed in terms of power laws [3-9]. Two creep regimes are evidenced. In the low stress range, diffusional creep (Nabarro-Herring or Coble mechanism) is usually invoked, whereas at higher stresses dislocational creep is reported.

In addition to the analysis of the macroscopic mechanical behavior, microstructural evolutions after deformation were studied by means of Scanning Electron Microscopy (SEM) and/or Transmission Electron Microscopy (TEM) examinations [5,8]. Numerous dislocation dipoles were found in the case of single slip [10]. In double slip conditions, sub-boundaries made of hexagonal networks of screw dislocations were reported [11]. Recently, Electron Back Scattered Diffraction (EBSD) was also applied to characterize the microstructure of crept $\mathrm{UO}_{2}$ pellets [12-14].

All these examinations point out a sub-structuration process 
within $\mathrm{UO}_{2}$ grains in the dislocational creep regime. Plastic deformation (or visco-plastic one, in the case of ceramics such as uranium dioxide) is accompanied by the creation of dislocations. Under a sufficient temperature, these dislocations glide and climb and reorganize into sub-boundaries within the original grains. This mechanism is often called "dynamic recovery" or "polygonization" $[15,16]$. At low or moderate deformation levels, hardening processes (by increasing the number of dislocations) and dynamic recovery (by migration and organization of these dislocations in networks or walls) operate simultaneously. At higher deformation rates, some sub-boundaries progressively rotate to form new grains. This phenomenon, frequently observed in metals and also in rocks, is referred to "sub-grain rotation recrystallization" [17-19]. It is favored when the subgrain boundaries contain small precipitates, which pin them in place.

Nowadays, EBSD offers the possibility to characterize this grain sub-structuration and quantify its evolution according to the deformation conditions. However, few studies concern the deformation by creep. Among the numerous studies on creep deformation of $\mathrm{UO}_{2}$, none of them gives quantified results about substructure development as a function of deformation rate and level. Indeed, most of the disorientations induced by creep are very low, mostly below the angular resolution of about $1^{\circ}$ of standard EBSD acquisitions.

This work is aimed to quantify the sub-structured microstructure induced by dislocational creep in $\mathrm{UO}_{2}$, as a function of strain level and strain rate. Stoichiometric $\mathrm{UO}_{2}$ pellets were mechanically tested in uniaxial compression conditions at $1500^{\circ} \mathrm{C}$ at different strain rates (from $\sim 5 \times 10^{-6} \mathrm{~s}^{-1}$ to $70 \times 10^{-6} \mathrm{~s}^{-1}$ ) and strain levels (from $3 \%$ to $11 \%$ ) in the secondary creep regime [20]. EBSD data were acquired in improved angular resolution mode [21]. The quantification of the grain sub-structuration was achieved by applying the methodology proposed in Ref. [14]. It is based on an optimized protocol for EBSD data acquisition and processing with the potential to detect and quantify low angle sub-boundaries disoriented down to $0.1^{\circ}$ in $\mathrm{UO}_{2}$. In addition, Accurate-ECCI experiments were also performed to image the dislocations induced by creep and their arrangement in very low sub-grain boundaries $[22,23]$. Moreover, an advanced sub-grain completion procedure was applied to the EBSD data to quantify the sub-grain size evolution [24]. Finally, Geometrically Necessary Dislocations (GNDs) densities were calculated using the ATEX software [14,25]. All these EBSD-based quantities allow to quantify the substructure evolution of crept $\mathrm{UO}_{2}$ pellets, as a function of strain level and strain rate.

\section{Experimental}

\subsection{Materials}

Cylindrical pellets were made from a single batch of $\mathrm{UO}_{2}$ powder by uniaxial pressing under $450 \mathrm{MPa}$. They were further sintered at $1780 \pm 20{ }^{\circ} \mathrm{C}$ during $5,5 \mathrm{~h}$, under $\mathrm{Ar}+5 \% \mathrm{H}_{2}$ atmosphere (to ensure their stoichiometry). Their final density measured by an immersion method was of $98.1 \%$ of the $\mathrm{UO}_{2}$ theoretical one. The pellets had a diameter of $8 \mathrm{~mm}$ and a height of $15 \mathrm{~mm}$. This height is slightly higher than that of standard pellets for PWRs $(12-13 \mathrm{~mm})$ in order to favor a better distribution of the deformation during uniaxial compression tests.

The microstructure of the as sintered reference sample (not deformed) is shown in Fig. 1. This SEM micrograph was taken in backscattered electron (BSE) imaging mode, in order to reveal the grains by orientation contrast. The grains are equiaxed in shape and have a size of about $15 \mu \mathrm{m}$. Small round fabrication voids are observed in intra- and inter-granular locations. The surface fraction of these voids was evaluated at $1.7 \%$ by image analysis using the

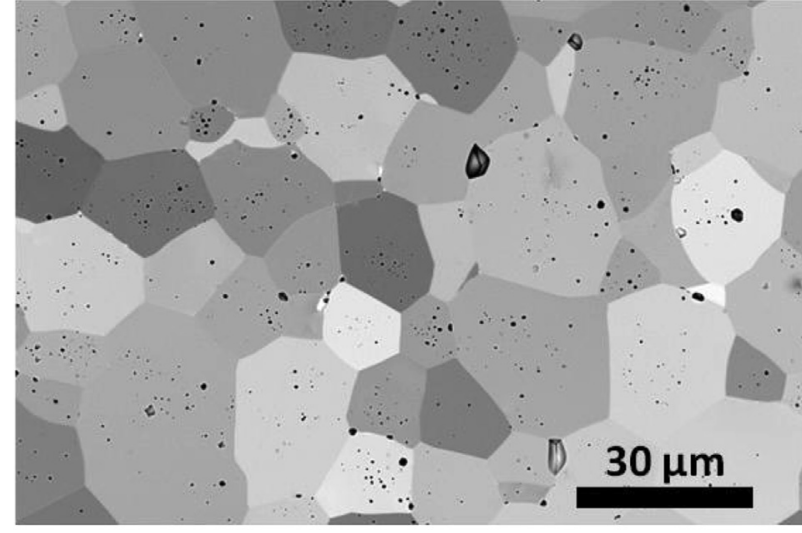

Fig. 1. SEM micrograph in BSE mode acquired on the reference sample R (as sintered).

AnalySIS image software [13,14]. This value is slightly different from that expected from density measurements (i.e. 1.9\%), probably because of image sampling effects.

\subsection{Compression creep tests}

Uniaxial compression creep tests were performed in an adapted furnace on a screw-type Instron compression test machine. All these tests were conducted at $1500{ }^{\circ} \mathrm{C}$ and under the same atmosphere as that used for sintering $\left(\mathrm{Ar}+5 \% \mathrm{H}_{2}\right)$, to maintain their stoichiometry. The compression tests conditions were chosen to deform pellets in the dislocational creep regime (at high stress). They were performed by displacing the mobile sleeper of the machine at an imposed constant speed. Pellets were fixed between two tungsten plates and the deformation was measured with two extensometers placed in contact with these plates. Complementary details concerning the testing machine characteristics can be found in Refs. $[8,13,20]$. The cooling rate, after all tests, was $17^{\circ} \mathrm{C}$ per min. During this step, a weak force (less than $250 \mathrm{~N}$ ) remained applied to hold the pellet in place. Potential microstructure evolution induced by the cooling procedure was evaluated. For this purpose, an additional pellet was directly quenched after deformation (a cooling rate of about $200{ }^{\circ} \mathrm{C} . \mathrm{min}^{-1}$ was achieved between $1500^{\circ} \mathrm{C}$ and $900^{\circ} \mathrm{C}$, that gradually decreased after) [20]. The grain substructuration obtained after quenching was similar to the one obtained with a cooling rate of $17^{\circ} \mathrm{C} \cdot \mathrm{min}^{-1}$ (of course for similar deformation conditions). This result confirms that our cooling procedure did not significantly modify the deformed microstructure.

Nine pellets (referenced D1 to D9) were tested in order to study the effect of the strain level and strain rate on the grain substructuration. Table 1 summarizes the main deformation conditions of all pellets (including the reference one $\mathrm{R}$ (not deformed)). The pellets [D1 to D5] form a first group deformed under the same strain rate of $20 \times 10^{-6} \mathrm{~s}^{-1}$ to reach different strain levels ranging from $3 \%$ to $11 \%$. The pellets [D3, D6, D7] and the [D5, D8, D9] ones form the second and the third groups respectively: they were deformed under different strain rates ranging from $5 \times 10^{-6} \mathrm{~s}^{-1}$ to $40-70 \times 10^{-6} \mathrm{~s}^{-1}$ to reach a strain level of about 7 and $10-11 \%$ respectively.

\subsection{Characterization methods}

Pellets were sectioned in their central part, parallel to the compression axis, with a diamond wire saw. To get a suitable surface quality for EBSD and ECC imaging, the samples were 
Table 1

Main deformation conditions of the $\mathrm{UO}_{2}$ pellets.

\begin{tabular}{|c|c|c|c|}
\hline Pellet designation & Strain rate $x 10^{-6}\left(\mathrm{~s}^{-1}\right)$ & Strain level (\%) & Duration of the test \\
\hline $\mathrm{R}$ & 0 & 0 & 0 \\
\hline D1 & 20 & 3 & $37 \mathrm{~min}$ \\
\hline D2 & 20 & 5.1 & $1 \mathrm{~h}$ \\
\hline D3 & 20 & 7 & $1 \mathrm{~h} 19 \mathrm{~min}$ \\
\hline D4 & 20 & 9.4 & $1 \mathrm{~h} 28 \mathrm{~min}$ \\
\hline D5 & 20 & 11 & $1 \mathrm{~h} 41 \mathrm{~min}$ \\
\hline D6 & 5 & 7 & $5 \mathrm{~h} 20 \mathrm{~min}$ \\
\hline D7 & 40 & 7 & $20 \mathrm{~min}$ \\
\hline D8 & 5 & 10 & $6 \mathrm{~h} 10 \mathrm{~min}$ \\
\hline D9 & 70 & 11 & $28 \mathrm{~min}$ \\
\hline
\end{tabular}

mechanical polished in three main steps: a first grinding with silicon carbide discs (grit from 800 to 4000), a diamond polishing with a $1 \mu \mathrm{m}$ polycrystalline suspension and a final polishing with a $0.02 \mu \mathrm{m}$ non-crystallizing colloidal silica suspension. The pellets nomenclature (given in Table 1) was also applied to the corresponding samples. In each sample, the most deformed area was identified by SEM examinations and corresponded systematically to the central part of the pellet. Fig. 2 illustrates the microstructure obtained after compression test for samples D7 and D9. Significant variations in the BSE signal were observed inside the prior grains and evidenced sub-grains induced by the plastic deformation. The grains remained essentially equiaxed even after large strains $(\sim 11 \%$, cf. Fig. 2b). Damages in the form of inter-granular cavities, have been observed in certain previous studies performed on $\mathrm{UO}_{2}$ pellets with grain sizes varying from 9 to $36 \mu \mathrm{m}[8,12]$. In this study, such damages were only observed at the highest strain rate and level (sample D9), in the central part of the pellet, as illustrated in Fig. 2b (red arrows). These cavities are globally elongated in a direction parallel to the compression axis, in accordance with results given in Refs. [8,12].

EBSD maps were acquired in a FEI NovaNano SEM (with an accelerating voltage of $20 \mathrm{kV}$ and at a working distance of $15 \mathrm{~mm}$ ) equipped with a Nordlys Nano II camera from Oxford Instruments (in $2 \times 2$ binning mode $-672 \times 512$ pixels). The "refined accuracy mode" in AZTEC was used to improve the angular resolution of the EBSD orientation data [21]. Large EBSD maps $(500 \times 344 \mu \mathrm{m})$ were acquired with a step of $0.4 \mu \mathrm{m}$, on the reference and on the deformed samples (in the most deformed area corresponding to the central part of the pellets). Each EBSD map covered around 1000 grains and was large enough to be representative of the deformed state of the pellets, as checked in Ref. [20]. The indexing rate of EBSD data was more than $98 \%$.

These EBSD maps were used to analyze and quantify the sub- boundaries induced by the deformation. For this purpose, the orientation data were processed according to the optimized procedure proposed in Ref. [14]. It allows to efficiently reveal subboundaries disoriented down to $0.1^{\circ}$ in $\mathrm{UO}_{2}$ crept samples and includes two main steps: data cleaning and data filtering. The cleaning step was adapted for porous material. Indeed, noisy orientations are often measured by EBSD over the voids. They need to be removed from the data set to quantify properly low disorientations induced by creep. Then, the orientation noise of the EBSD data can be further reduced by applying a Kuwahara filter. This filter reduces the orientation noise (estimated to reach locally maximal values of $0.3^{\circ} / 0.5^{\circ}$ for our acquisition conditions), while preserving true continuous disorientation lines of the same order of magnitude.

Three EBSD-based parameters were calculated from each EBSD map to quantify the substructure induced by creep and its evolution with deformation conditions:

(1) The linear fraction of sub-boundaries was quantified, considering different disorientation classes. The critical disorientation angle chosen to define a grain boundary was set at $5^{\circ}$. Thus, hereafter, the term "grain boundary" (GB) refers to all lines of angular disorientation greater than or equal to $5^{\circ}$. The term "Sub-grain boundary or sub-boundary (S-GB)" refers to all lines of angular disorientation lower than $5^{\circ}$ which appear inside the prior grains during creep deformation. On the large scale EBSD maps presented in this work, the disorientations below $0.25^{\circ}$ were not quantified. Indeed, the experience showed that these very low disorientations were not only associated to sub-boundaries induced by creep, but also to mechanical polishing defects of the sample.

(2) The network of sub-boundaries was further transcripted in GNDs and the evolution of GNDs density with creep

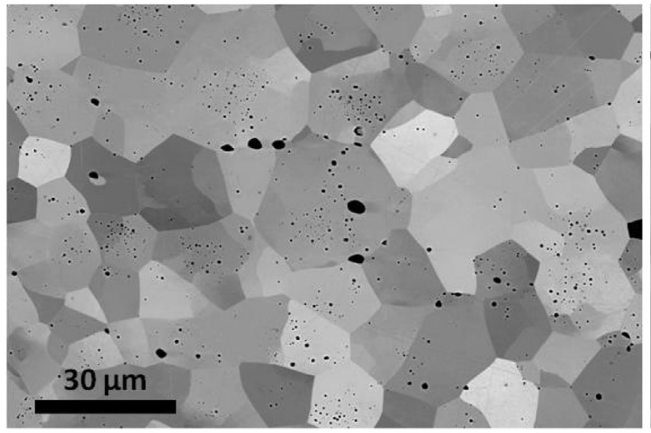

(a)

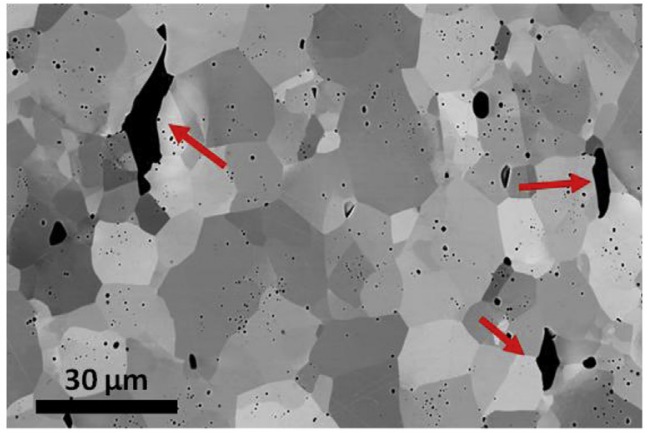

(b)

Fig. 2. SEM micrographs in BSE mode acquired in the deformed samples (a) D7 and (b) D9. Compression direction: vertical. 


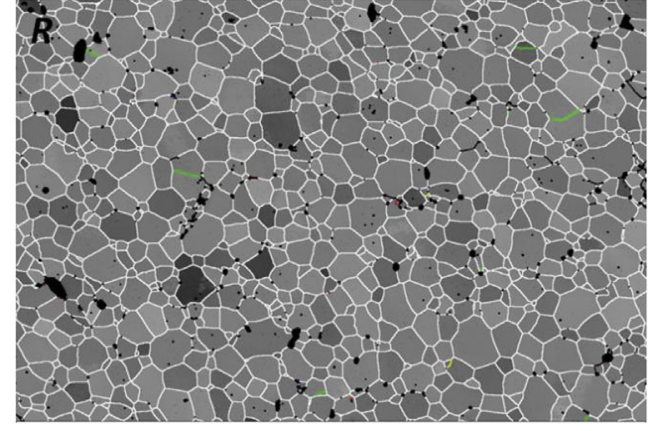

(a)

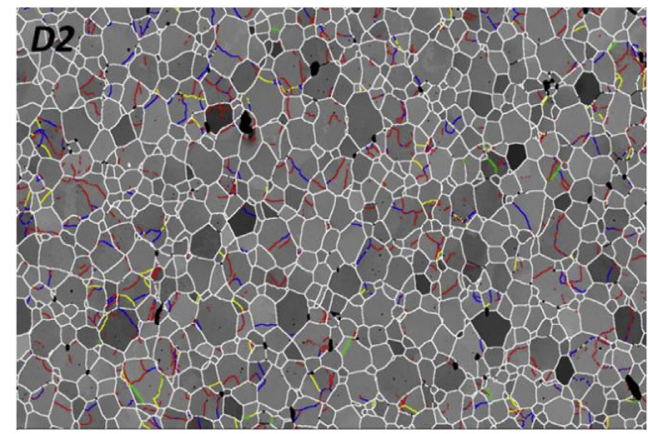

(c)

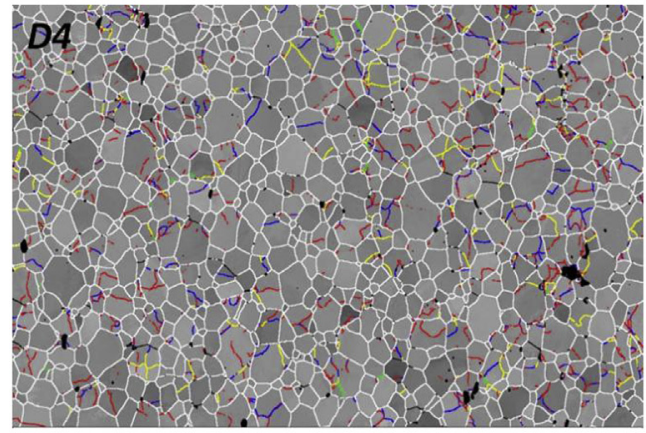

(e)

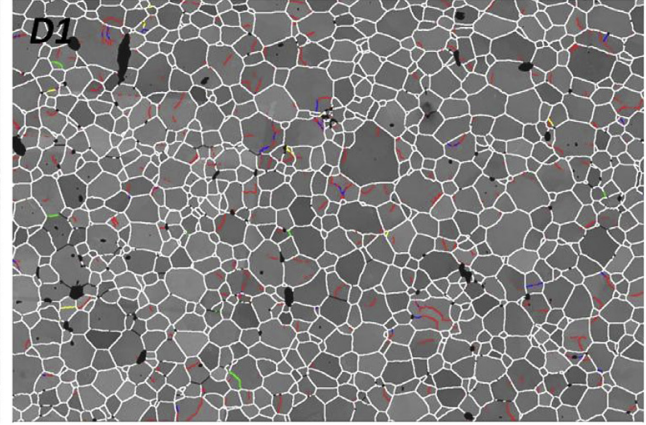

(b)

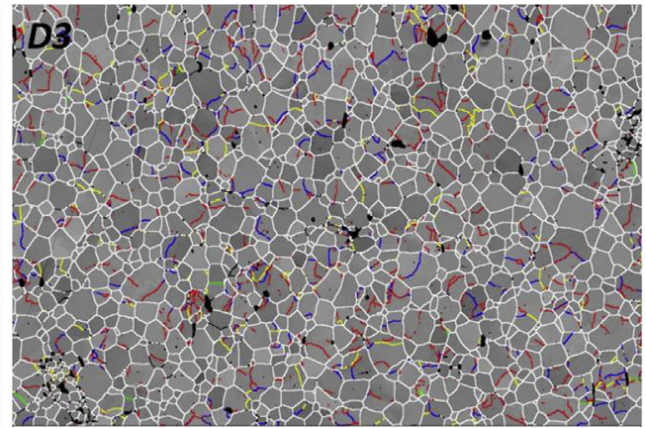

(d)

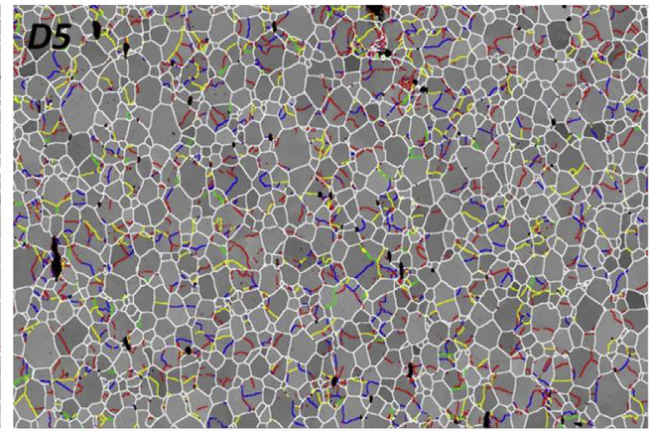

(f)

$\rightarrow$ Compression direction

Disorientation angle $\theta$

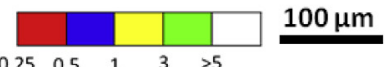

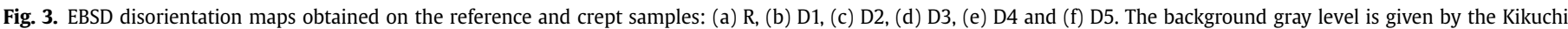
Pattern Quality index. Non-indexed points, mostly found over the fabrication porosity, are black. The compression direction is horizontal as indicated by the black arrows.

deformation was quantified from all the EBSD maps (filtered data). The calculations were performed with ATEX software [25], according to the method described in Ref. [14]. It is based on the evaluation of the Nye dislocation tensor from the lattice curvature measured by EBSD. The total dislocation density is then expressed in terms of the entrywise 1-norm of the Nye tensor. In this work, the limit angle chosen to translate a misorientation into GNDs was $5^{\circ}$. The result of this calculation gives the density distribution of GNDs over the EBSD mapped region from which the average density of GND's can be evaluated.

(3) The sub-grains delimited by low disorientation boundaries were detected by an advanced sub-grain detection procedure $[14,24]$ and the evolution of their average size according to the strain level was quantified. The classical EBSD grain recognition procedure clusters pixels disoriented from their neighbors by less than a threshold angle $\omega_{0}$. The present procedure proceeds in two steps and defines an upper and lower bound for the threshold angle. The upper value is set higher than the orientation noise (here it was set to $0.25^{\circ}$ ). It allows, in the first step, to identify a primary network of wellidentified grains and sub-grains. However, inside those grains, $0.25^{\circ}$ misoriented lines can be detected but they do not mark out closed sub-grains. Thus, the second step aims to close these open lines by tracking a path between neighboring pixels considering disorientation angles down to the lower bound (here it was set to $0^{\circ}$ ).

Finally, Electron Contrast Channeling Image (ECCI) experiments were performed on the deformed microstructures, to contrast the dislocations induced by creep and their progressive arrangement in sub-boundaries. All ECC images were acquired with a Zeiss Auriga 40 FEG-SEM equipped with a GEMINI-type electron column. The images were acquired at a working distance of $7 \mathrm{~mm}$, an acceleration voltage of $20 \mathrm{kV}$ and a beam current of $550 \mathrm{pA}$ (with the $30 \mu \mathrm{m}$ center aperture). Details for the acquisition steps of these images 


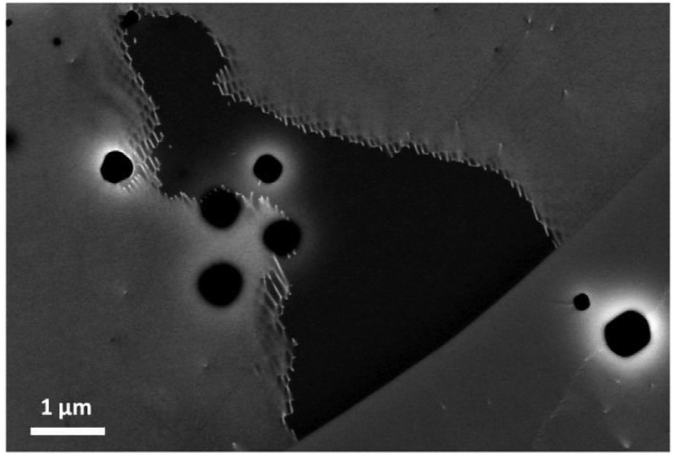

(a)

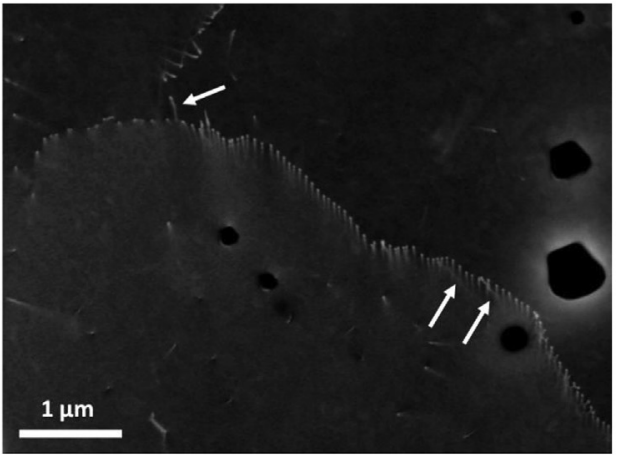

(b)

Fig. 4. ECC images acquired on samples (D1) and (D3). The white arrows point mobile dislocations interacting with sub-boundary dislocations.

are given in Refs. [14,22,23,26,27].

\section{Results}

\subsection{Influence of strain level on the grain sub-structuration}

Our results show that creep deformation induced a pronounced network of sub-boundaries inside the prior $\mathrm{UO}_{2}$ grains. With increasing strain level, the sub-boundaries were observed to extend inside the grains and to increase their disorientations. This is illustrated in Fig. 3 that compares the EBSD maps performed on the reference and the first group of pellets (D1 to D5). Deformed pellets were tested with a strain rate of about $20 \times 10^{-6} \mathrm{~s}^{-1}$ up to an average deformation level ranging from $3 \%$ to $11 \%$. On each EBSD map, the white lines correspond to the prior grain boundaries with disorientation angles larger than $5^{\circ}$. The other colored lines correspond to sub-boundaries, with disorientation indicated by the attached color code.

After 3\% of deformation, a network of very low angle boundaries $\left(<1^{\circ}\right.$, in blue and red) started to develop inside the prior $\mathrm{UO}_{2}$ grains (Fig. 3b). It was obviously absent within the grains of the reference sample; the few green lines in Fig. 3a corresponding to scarce low angle grain boundaries, with typical disorientations between $3^{\circ}$ and $5^{\circ}$. With increasing strain level (Fig. $3 c-f$ ), these subboundaries were observed to extend inside the prior grains, new very low angle boundaries comprised between $1^{\circ}$ and $5^{\circ}$ (yellow and green lines) being created continuously. Interestingly, already existing very low angle boundaries progressively reached higher disorientations, as evidenced in Fig. $3 \mathrm{c}-\mathrm{f}$.

The ECC images given by Fig. 4 show the dislocations created inside the grains during the deformation. After 3\% of deformation, these dislocations were already organized in a regular network to form very low angle sub-boundaries (see Fig. 4a). As most of these sub-boundaries had very low disorientations, boundary dislocations could be individually distinguished by ECCI. This is consistent with the EBSD analysis that revealed mainly sub-boundaries disoriented by less than $0.5^{\circ}$ at this deformation level (cf. Fig. $3 \mathrm{~b}$ ). At higher strain level, more and more sub-boundaries were observed for which the individual dislocations could no longer be distinguished by ECCI. Mobile dislocations were also observed within the grains, some interacting with sub-boundary dislocations, disturbing the regular network, as illustrated in Fig. $4 \mathrm{~b}$ (see the white arrows). These ECCI observations confirm the progressive increase in sub-boundary disorientations with the strain level, previously deduced from EBSD maps.

\subsection{Quantitative microstructure evolution with the strain level}

The sub-boundaries networks were further quantified by calculating the linear fractions of boundaries and sub-boundaries according to the disorientation classes they belong to. Fig. 5a considers all disorientations higher than $0.25^{\circ}$ and classifies them in

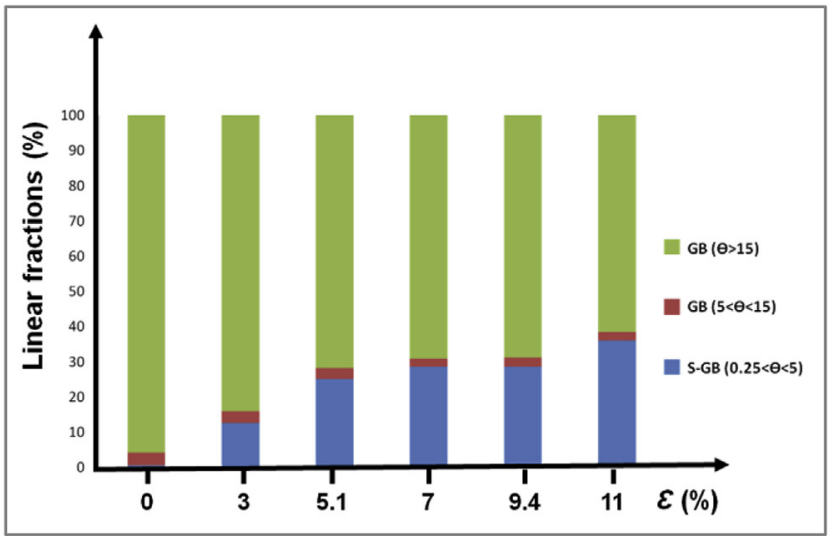

(a)

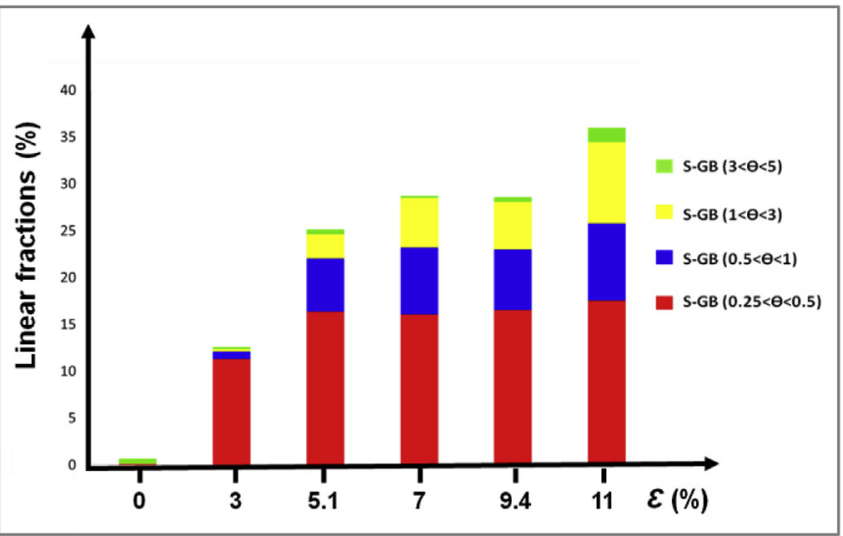

(b)

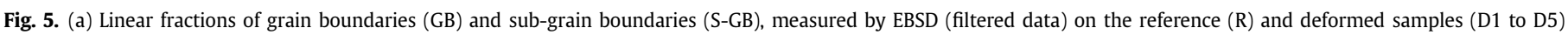
(considering all disorientation lines with angles $>0.25^{\circ}$ ), (b) close-up view of the sub-grain boundaries. 


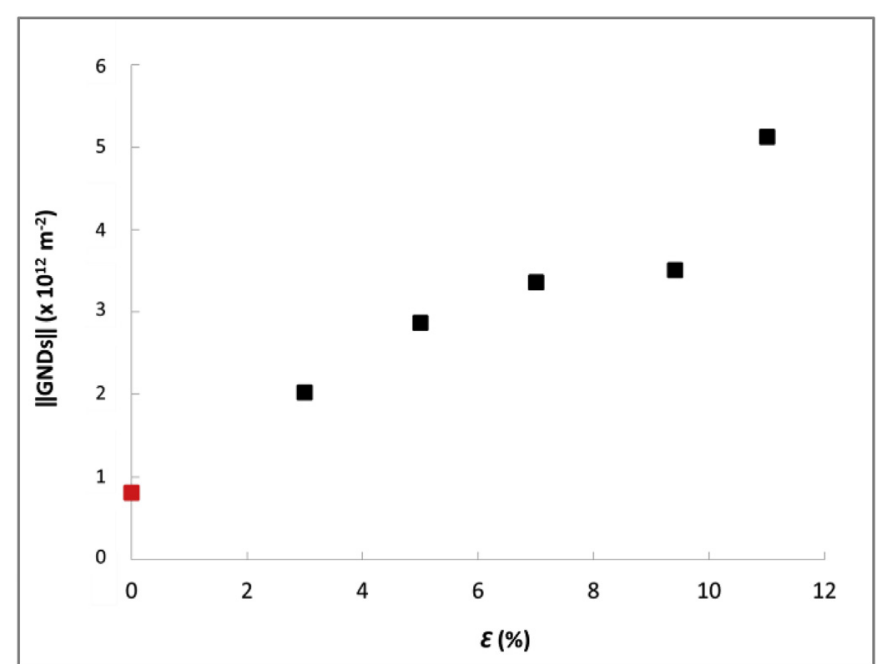

Fig. 6. Evolution of the average GNDs densities $\left\langle\rho_{\mathrm{GND}}\right\rangle$ with the strain level $(\varepsilon)$ for the reference (R, red marker) and deformed samples (D1 to D5, black markers). (For interpretation of the references to color in this figure legend, the reader is referred to the Web version of this article.)

three groups: sub-boundaries with disorientations comprised between $0.25^{\circ}$ and $5^{\circ}$, boundaries with disorientations comprised between $5^{\circ}$ and $15^{\circ}$ and high angle boundaries disoriented by more than $15^{\circ}$. Fig. $5 \mathrm{~b}$ considers only the sub-boundaries and classifies them in four classes (the same classification and color code as that used in Fig. 3 is applied).

In the reference sample (R), $96 \%$ of the measured disorientations were greater than $15^{\circ}$. Only $0.7 \%$ of the considered disorientations were lower than $5^{\circ}$. After only $3 \%$ of deformation (D1), the fraction of sub-boundaries with disorientations lower than $5^{\circ}$ reached $13 \%$, among which $12 \%$ were disoriented by less than $1^{\circ}$. This subboundaries total fraction reached $25 \%$ after a deformation of $5.1 \%$ (sample D2).

Between $5.1 \%$ and $9.4 \%$ of deformation (samples D2, D4), the linear fraction of sub-boundaries increased only slightly. The apparent saturation of sub-boundaries could be related to the establishment of quasi-stationary conditions between creation and annihilation of dislocations.

After $11 \%$ of deformation (sample D5), the linear fraction of subboundaries increased again, especially those belonging to the yellow and green colored classes (with disorientation angles comprised respectively between $1^{\circ}$ and $3^{\circ}$, and between $3^{\circ}$ and $5^{\circ}$ ) (Fig. 5b). The lower classes of disorientation (less than $1^{\circ}$ ) also increased but less markedly. All these results tend to show that at high strain level ( $11 \%$ in this case), new sub-boundaries with very low disorientation angles (cf. classes $\left[0.25^{\circ}{ }_{-} 0.5^{\circ}\right.$ [ and $\left[0.5^{\circ} 1^{\circ}\right.$ ) are formed, while the disorientation angle of already existing subboundaries increases (cf. classes $\left[1^{\circ} 3^{\circ}\left[\right.\right.$ and $\left[3^{\circ} 5^{\circ}[\right.$ ).

The evolution of the average density of GNDs with creep deformation was quantified from the filtered EBSD maps of samples $\mathrm{R}$ and $\mathrm{D} 1$ to D5. The result is given in Fig. 6. It shows the same evolution with the strain level as that previously evidenced for the linear fraction of the sub-boundaries (cf. Fig. 5). From 0 to $5.1 \%$ of deformation, the average GNDs density increases from $0.8 \times 10^{12} \mathrm{~m}^{-2}$ to $3.5 \times 10^{12} \mathrm{~m}^{-21}$ then it is more or less stable up to $9.4 \%$ of deformation and finally increases again up to

\footnotetext{
1 The density calculated on non deformed sample $\mathrm{R}$ corresponds to the orientation noise. This noise is converted in a certain level of dislocation density, often called spurious density $\left(\rho_{\mathrm{sp}}\right)[14]$.
}

$5.1 \times 10^{12} \mathrm{~m}^{-2}$ at $11 \%$ of deformation.

The EBSD data were further processed to detect the sub-grains formed inside the prior grains and quantify the evolution of their average size with the strain level (see section 2.3 for further details). Fig. 7a illustrates the processing for sample D5; it highlights in random colors sub-grains identified within the prior $\mathrm{UO}_{2}$ grains contoured in white. Sub-boundaries form a relatively homogeneous network, even if they sometimes tend to concentrate close to the grain boundaries. Fig. 7b shows that the initially about $15 \mu \mathrm{m}$ size grains are fragmented into sub-grains, with a decreasing average size with increasing strain level. This average size reaches around $11 \mu \mathrm{m}$, between $5.1 \%$ and $9.4 \%$ of deformation, and drops around $9 \mu \mathrm{m}$ at a deformation level of $11 \%$. These results are fully consistent with those obtained for sub-boundaries: the more the number of sub-boundaries increases, the more the average size of the sub-grains decreases.

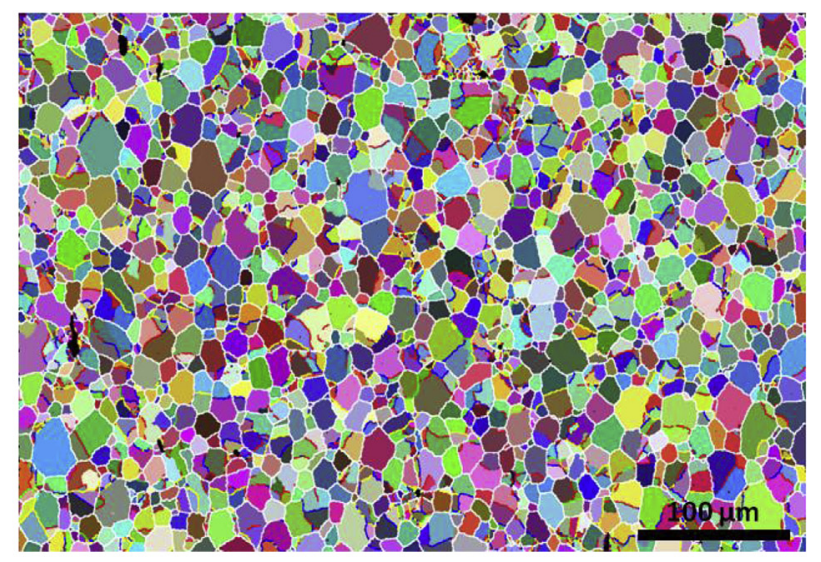

(a)

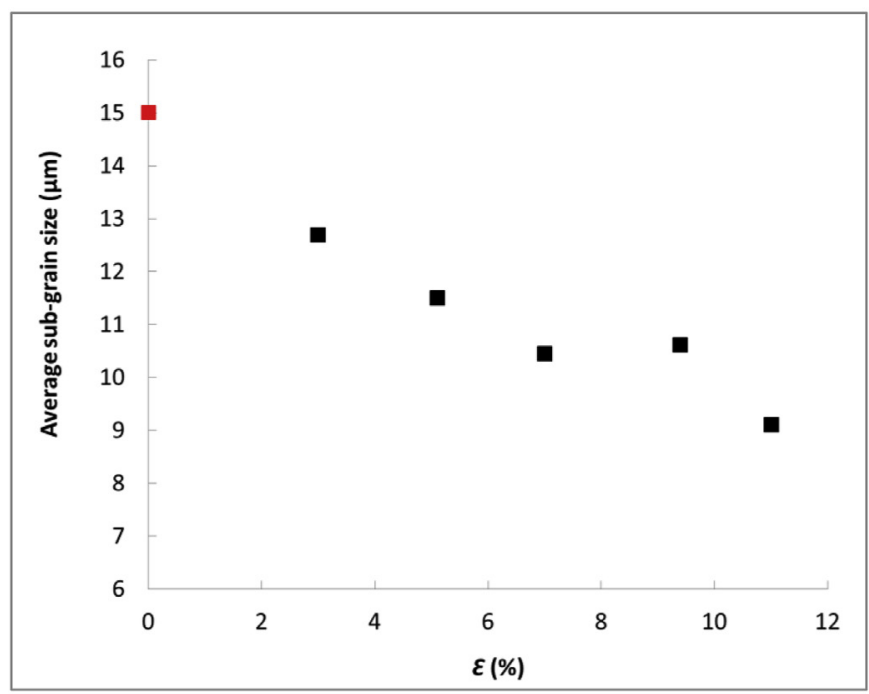

(b)

Fig. 7. (a) Disorientation map of sample D5 (Fig. 3f) showing in random colors subgrains identified within the prior $\mathrm{UO}_{2}$ grains contoured in white, (b) Evolution of the mean sub-grain size with the strain level $(\varepsilon)$ for the reference $(\mathrm{R})$ and deformed samples (D1 to D5) (the red marker corresponds to the prior $\mathrm{UO}_{2}$ grain size). (For interpretation of the references to color in this figure legend, the reader is referred to the Web version of this article.) 


\subsection{Influence of strain rate on the grain sub-structuration}

The two groups of pellets (D3, D6, D7) and (D5, D8, D9) were deformed under different strain rates ranging from $5 \times 10^{-6} \mathrm{~s}^{-1}$ to $40-70 \times 10^{-6} \mathrm{~s}^{-1}$ to reach a mean strain level of about 7 and $11 \%$ respectively. Their study allows analyzing and quantifying the influence of the strain rate on the grain sub-structuration.

As shown by Fig. 8, the linear fraction of sub-boundaries increases with the strain rate, in each disorientation class $\left(\left[0.25^{\circ} \_0.5^{\circ}\right.\right.$ [, $\left[0.5^{\circ} 1^{\circ}\left[\right.\right.$ and $\left[1^{\circ} 3^{\circ}[)\right.$ for both groups. No "saturation" of the population of sub-boundaries, whatever their disorientation class, is observed. This result points out a continuous formation of new sub-boundaries within the original grains. Moreover, at high strain level $(11 \%)$, the fraction of sub-boundaries disoriented between $\left(1^{\circ}\right.$ and $5^{\circ}$ ) (colored in yellow and green in Fig. 8b) increases markedly at high strain rates $\left(70.10^{-6} \mathrm{~s}^{-1}\right.$ ) (from $10 \%$ (D5) to $13 \%$ (D9)). This result confirms that the $\mathrm{S}-\mathrm{GB}$ disorientation angles increase with deformation, leading to the creation of new grain boundaries.

The evolution of the average dislocation densities with strain rate was also quantified as well as the evolution of the mean subgrain size. The GNDs density increased with the strain rate whereas the mean sub-grain size decreased. This is illustrated in Fig. 9. For example, the prior $\mathrm{UO}_{2}$ grains, with an average size of $15 \mu \mathrm{m}$, were fragmented into sub-grains of about $8 \mu \mathrm{m}$ at $11 \%$ of deformation at a strain rate of $70 \times 10^{-6} \mathrm{~s}^{-1}$.

\section{Discussion}

The evolution of the crept microstructure of $\mathrm{UO}_{2}$ was studied as a function of the strain level and rate, thanks to an optimized methodology for EBSD data acquisition and post-processing. The progressive development of sub-boundaries networks within the grains was clearly evidenced and confirmed by ECC images.

Moreover, the results show that our methodology is efficient to quantify the increase of linear fraction of the sub-boundaries and the GNDs density, with increasing strain level (see Figs. 5 and 6) and strain rate (see Figs. 8 and 9). At a strain rate of $\sim 20 \times 10^{-6} \mathrm{~s}^{-1}$, the linear fraction of the sub-boundaries ranges from almost zero to $\sim 25 \%$ for deformations comprised between $\sim 5 \%$ and $\sim 9.5 \%$. It increases again up to $\sim 35 \%$, when the deformation reaches $\sim 11 \%$. The corresponding GNDs density then reaches $5 \times 10^{12} \mathrm{~m}^{-2}$. When the strain rate is increased up to $\sim 70 \times 10^{-6} \mathrm{~s}^{-1}$, the two previous quantities reach $\sim 45 \%$ and $\sim 7.5 \times 10^{12} \mathrm{~m}^{-2}$ respectively (for a strain level of $11 \%)$. Concomitantly, the mean sub-grain size decreases progressively with the increase of strain rate and level. Also, new sub-boundaries disoriented by less than $1^{\circ}$ are continuously created whereas already existing sub-boundaries increase their disorientation. This evolution could potentially create new grains with boundaries disoriented by more than $5^{\circ}$ for higher deformation levels and rates. Thus, in the dislocational creep domain, $\mathrm{UO}_{2}$ is subject to a dynamic recovery mechanism by progressive rotation of sub-grains (in other words, by progressive increase in subboundaries disorientation angle) [18].

4.1 The sub-structuration process of $\mathrm{UO}_{2}$ seems to take place in three steps (cf. Figs. 6 and 7)

- a first one (between 0 and $~ 5 \%$ of deformation, at $\sim 20 \times 10^{-6}$ $\mathrm{s}^{-1}$ ), in which more and more numerous dislocations appear and form sub-boundaries,

- a second one (between $\sim 5 \%$ and $\sim 9.5 \%$, or slightly more), in which the sub-boundaries population is stabilized,

- a third one (around $11 \%$ or more, or at high strain rates), in which the sub-boundaries and GNDs densities increase again.

This evolution according to three steps could be surprising as all the tests were performed in the macroscopic secondary/stationary creep regime, in which an equilibrium between the creation and the annihilation of dislocations would be expected (as only observed in the second step described above). However, one has to keep in mind that the transition between the different creep regimes is progressive. Thus, it is reasonable to assume that the less deformed pellets $(\leq 5 \%)$ were still in a transient regime between primary and secondary creep. Conversely, the more deformed ones could be close to the transition to the ternary creep regime (especially because all observations were performed in the most deformed areas of the pellet).

The systematic quantification of sub-boundary linear fractions, GNDs density and sub-grain size, performed in this work for different testing conditions, offers the possibility to develop viscoplastic deformation models for $\mathrm{UO}_{2}$, at the microstructure scale, as initiated in Ref. [28]. This approach provides a real breakthrough in modelling the mechanical behavior of $\mathrm{UO}_{2}$, in comparison to more phenomenological ones (cf. [3,8], for example).

The development of a sub-structure within the $\mathrm{UO}_{2}$ prior grains, during compression tests at high temperature, as well as the influence of the testing conditions, have been little discussed in Literature. Dherbey et al. highlighted sub-boundaries in $\mathrm{UO}_{2}$ pellets, mechanically tested in conditions close to those of this study,

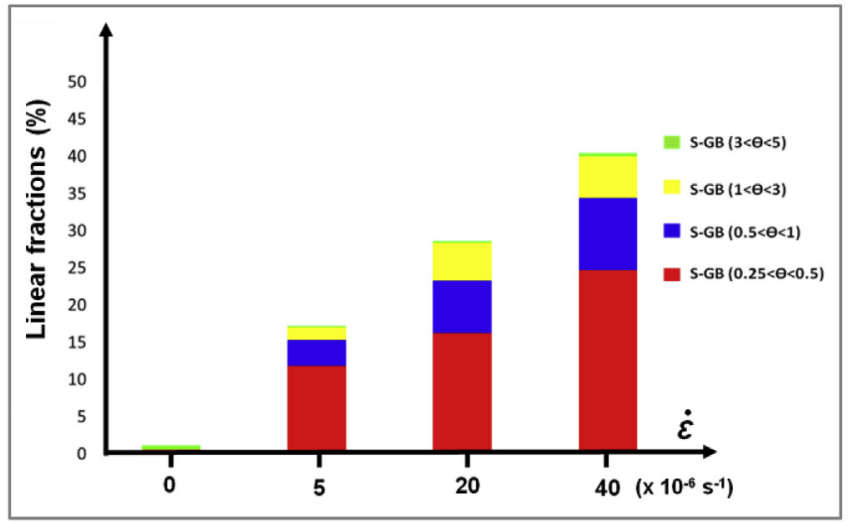

(a)

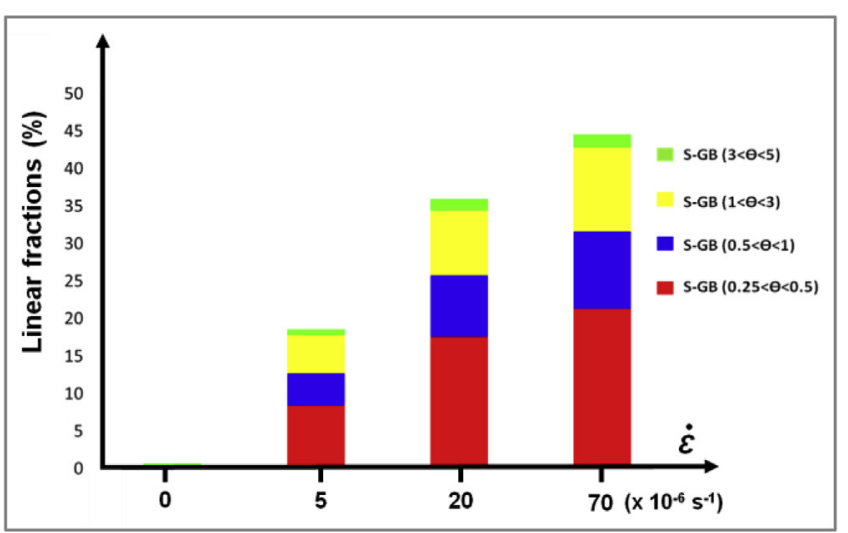

(b)

Fig. 8. Linear fractions of sub-grain boundaries, measured by EBSD (a) for pellets (R, D3, D6, D7) and (b) for pellets (R, D5, D8, D9). 


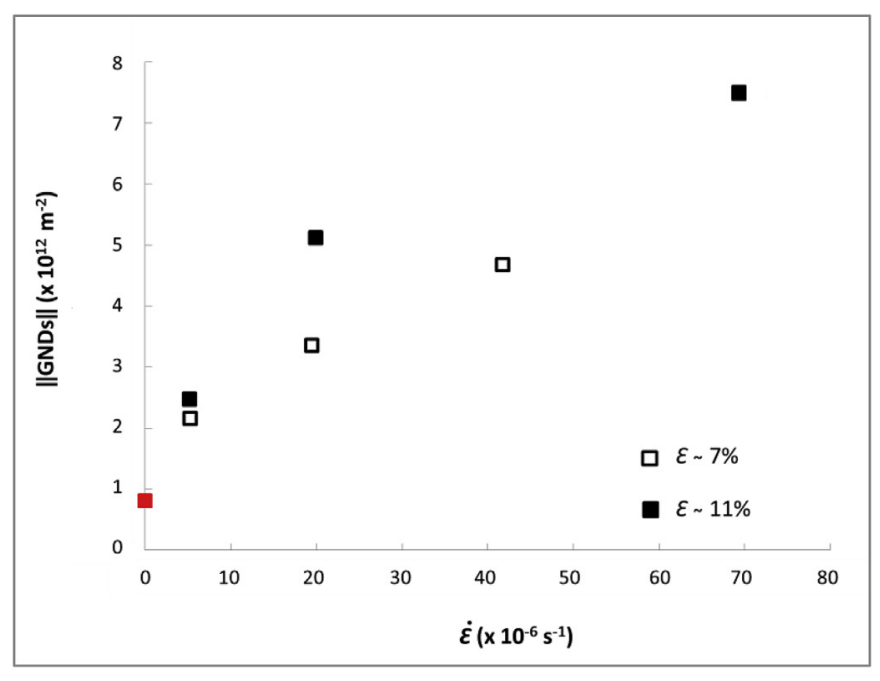

(a)

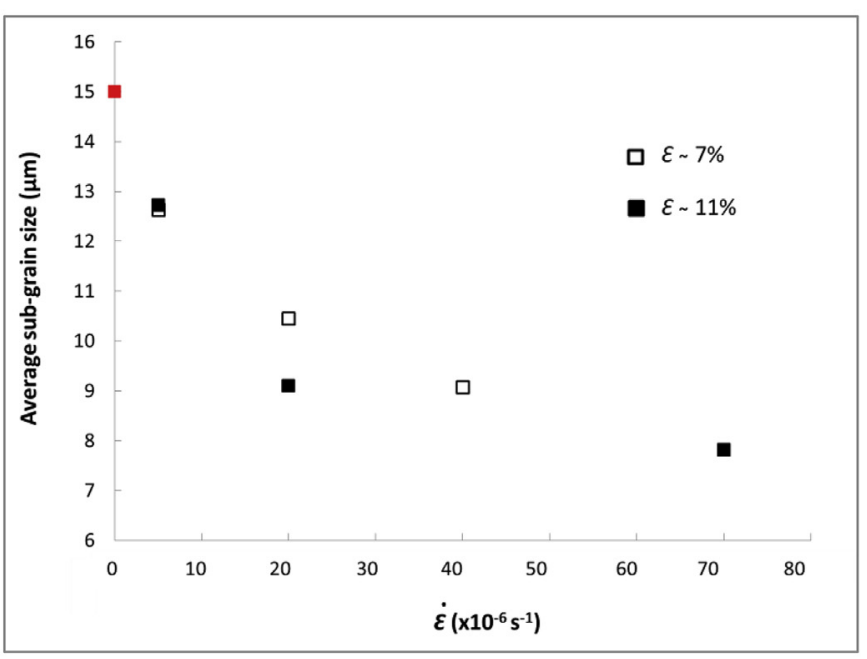

(b)

Fig. 9. (a) Average dislocation densities and (b) mean sub-grain size calculated from the EBSD maps of the two groups of pellets (D3, D6, D7) and (D5, D8, D9) respectively deformed to $7 \%$ and $11 \%$. The red marker corresponds to the reference pellet $\mathrm{R}$ (with no sub-grains). (For interpretation of the references to color in this figure legend, the reader is referred to the Web version of this article.)

thanks to an etching method [8]. This method did not allow any quantification and was perhaps not suitable for evidencing very low disorientations, contrary to EBSD and ECCI. These authors also performed TEM examinations on sub-boundaries but these observations were highly localized (as they were performed on thin foils) and did not produce any quantified data. The creep behavior of $\mathrm{UO}_{2}$ was described by a grain boundary sliding mechanism partially accommodated by dislocational creep within grains (giving rise to sub-boundaries formation). The fraction of the non-accommodated sliding by the intra-granular deformation caused the appearance of inter-granular cavities.

In the strain range applied in this work (3-11\%), small cavities have been observed only in the most deformed sample D9 (cf. Fig. 2b), whereas numerous ones were observed by other authors in pellets deformed under conditions similar to those used in the present work $[8,12]$. This difference could be related in part to the size of our pellets. Indeed, their height/diameter ratio (close to 2) was higher than standard ratios (close to 1.5) characterizing the pellets used in the other studies. This greater slenderness leads to a better distribution of the deformation and reduces its localization in the central zone of the pellet (which adopts a barrel shape, when uniaxially compressed). Recent examinations at CEA also pointed out a tendency to increase cavitation damage, with $\mathrm{UO}_{2}$ overstoichiometry [29]. It could be possible that our tests were carried out under better conditions of control of oxygen partial pressure, therefore of $\mathrm{UO}_{2}$ stoichiometry, than those performed in other studies.

Our results can be interestingly compared with those obtained on geological materials with structure similar to that of $\mathrm{UO}_{2}$ (ionic crystals, some with cubic lattice) such as olivine, monocrystalline $\mathrm{MgO}$, polycrystalline $\mathrm{NaCl}$, polycrystalline calcite $\left(\mathrm{CaCO}_{3}\right)$ [17-19,30-34]. All these studies cover higher strain levels, compared to the present work on $\mathrm{UO}_{2}$; this makes the quantification of deformation-induced sub-boundaries easier. However, the number of samples characterized was lower and the smallest disorientation angle detected by EBSD did not fall below $0.5^{\circ}$. Finally, the EBSD maps were performed on reduced areas of the deformed samples. All these factors limit the quantification of the sub-structuration process.

Despite these limitations, most of these studies confirmed that the number of sub-boundaries and their disorientation angle increased with the deformation. Particularly, Mariani et al. analyzed by EBSD the dynamic evolution of the microstructure of MgO monocrystals, tested in uniaxial compression between 1300 and $1500^{\circ} \mathrm{C}$ up to high deformation levels (more than 20\%) [17]. They concluded that dynamic recovery by sub-grains rotation was the main deformation mechanism of $\mathrm{MgO}$ single crystals. This phenomenon was also observed in the case of polycrystalline $\mathrm{NaCl}$, in which the average disorientation within grains increased with deformation, for tests performed at $165^{\circ} \mathrm{C}$ with deformation levels ranging from $7 \%$ to $54 \%$ [31]. The rate of this increase diminished at high strain levels. Then, new grains appeared in the original ones. The appearance of new grains was also evidenced in $\mathrm{CaCo}_{3}$ during deformation, by progressive increase of the SG-B disorientation angle [34]. It would be interesting to perform compression tests and characterizations on more deformed $\mathrm{UO}_{2}$ pellets (provided that cavities will not be too numerous), to check if a recrystallization by a sub-grain rotation process would also take place in this material.

\section{Conclusion and prospects}

The present paper quantifies the grain sub-structuration induced by dislocational creep in $\mathrm{UO}_{2}$, as well as its evolution as a function of strain level and strain rate. Stoichiometric $\mathrm{UO}_{2}$ pellets with $15 \mu \mathrm{m}$ mean grain size were deformed by uniaxial creep compression tests at $1500^{\circ} \mathrm{C}$ for different strain rates (from $5 \times 10^{-6} \mathrm{~s}^{-1}$ to $70 \times 10^{-6} \mathrm{~s}^{-1}$ ) and strain levels (from $3 \%$ to $11 \%$ ). Low and very low angle boundaries were induced by creep and formed sub-grains inside the prior $\mathrm{UO}_{2}$ grains.

The sub-boundaries disoriented down to $0.25^{\circ}$ were detected and quantified from large orientation maps thanks to an optimized methodology for EBSD data acquisition and post-processing. The results show that our methodology is efficient to quantify the subboundaries evolution inside the prior $\mathrm{UO}_{2}$ grains as a function of the deformation conditions. Three EBSD based quantities were calculated to follow this evolution: the linear fraction of the subboundaries, the corresponding GNDs density and the resulting mean sub-grain size. In addition, Accurate-ECCI technique was used to highlight the complex dislocation arrangement inside these creep induced sub-boundaries and confirmed the EBSD results.

It is shown that the linear fraction of sub-boundaries increases significantly with the strain level and rate. New very low angle 
boundaries disoriented by less than $1^{\circ}$ are continuously created whereas already existing sub-boundaries increase their disorientation. For higher deformation levels and rates, new grains with boundaries disoriented by more than $5^{\circ}$ could be potentially created. Thus, in the dislocational creep domain, $\mathrm{UO}_{2}$ is subject to a dynamic recovery (or even recrystallization) mechanism by progressive increase of sub-boundaries disorientation angle.

In a coming research, the same methodology will be applied to analyze the effect of microstructural parameters of $\mathrm{UO}_{2}$ pellets (such as voids distribution after sintering) on the creep deformed microstructure and its influence on the viscoplastic behavior of this ceramic.

\section{References}

[1] S. Aas, Mechanical interaction between fuel and cladding, Nucl. Eng. Des. 21 (1972) 237-253.

[2] B. Michel, J. Sercombe, C. Nonon, O. Fandeur, Modeling of pellet-cladding interaction, Compr. Nucl. Mater. 3 (2012) 677-712.

[3] M.S. Seltzer, J.S. Perrin, A.H. Clauer, B.A. Wilcox, Creep behavior of ceramic nuclear, React. Technol. 14 (1971) 99-135.

[4] B. Burton, G.L. Reynolds, J.P. Barnes, The influence of grain size on the creep of uranium dioxide, J. Mater. Sci. 8 (1973) 1690-1694.

[5] C.S. Yust, J.T.A. Roberts, On the observation of lattice and grain boundary dislocations in $\mathrm{UO}_{2}$ deformed at high temperature, J. Nucl. Mater. 48 (1973) 317-329.

[6] Y. Guérin, Etude par compression à hautes températures de la déformation plastique du bioxyde d'uranium polycristallin, J. Nucl. Mater. 56 (1975) $61-75$.

[7] D.B. Knorr, R.M. Canon, R.L. Coble, An analysis of diffusion and diffusional creep in stoichiometric and hyper-stoichiometric uranium dioxide, Acta Metall. 37 (1989) 2103-2123.

[8] F. Dherbey, F. Louchet, A. Mocellin, S. Leclercq, Elevated temperature creep of polycrystalline uranium dioxide: from microscopic mechanisms to macroscopic behaviour, Acta Mater. 50 (2002) 1495-1505.

[9] J.C. Gao, L.F. Wang, S.F. Wu, High-temperature creep properties of uranium dioxide pellet, T. NonFerr. Metals Soc. 20 (2010) 238-242.

[10] C.S. Yust, C.J. McHargue, Dislocation substructures in deformed uranium dioxide single crystals, J. Nucl. Mater. 31 (1969) 121-137.

[11] A. Alamo, J.M. Lefebvre, J. Soullard, Déformation plastique du bioxyde d'uranium: observation des sous-structures de dislocations, J. Nucl. Mater. 75 (1978) 145-153.

[12] X. Iltis, N. Gey, C. Cagna, A. Hazotte, Ph Sornay, Microstructural evolution of uranium dioxide following compression creep tests: an EBSD and image analysis study, J. Nucl. Mater. 456 (2015) 426-435.

[13] X. Iltis, M. Ben Saada, H. Mansour, N. Gey, A. Hazotte, N. Maloufi, A new characterization approach for studying relationships between microstructure and creep damage mechanisms of uranium dioxide, J. Nucl. Mater. 474 (2016) $1-7$.

[14] M. Ben Saada, N. Gey, B. Beausir, X. Iltis, H. Mansour, N. Maloufi, Subboundaries induced by dislocational creep in uranium dioxide analyzed by advanced diffraction and channeling electron microscopy, Mater. Char. 133
(2017) 112-121

[15] J. Robert-Amodeo, A review of experimental observations and theoretical models of dislocations cells and subgrains, Res. Mech. 23 (1988) 137-160.

[16] J.P. Poirier, Creep of Crystals : High-Temperature Deformation Processes in Metals, Ceramics and Minerals, Cambridge University Press, 1985.

[17] E. Mariani, J. Mecklenburgh, J. Wheeler, D.J. Prior, F. Heidelbach, Microstructure evolution and recrystallization during creep of $\mathrm{MgO}$ single crystals, Acta Mater. 57 (2009) 1886-1898.

[18] M.R. Drury, J.L. Urai, Deformation-related recrystallization processes, Tectonophysics 172 (1990) 235-253.

[19] M. Stipp, H. Stünitz, R. Heilbronner, S.M. Schmid, The eastern Tonale fault zone: a 'natural laboratory' for crystal plastic deformation of quartz over a temperature range from 250 to $700{ }^{\circ} \mathrm{C}$, J. Struct. Geol. 24 (2002) 1861-1884.

[20] M. Ben Saada, Etude du comportement visco-plastique du dioxyde d'uranium : Quantification par analyse EBSD et ECCI des effets liés aux conditions de sollicitation et à la microstructure initiale, PhD Thesis, Université de Lorraine, France, 2017.

[21] K. Thomsen, N.H. Schmidt, Improving the accuracy of orientation measurements using EBSD, Microsc. Microanal. 19 (Suppl 2) (2013) 724-725.

[22] H. Mansour, M.A. Crimp, N. Gey, N. Maloufi, Accurate electron channeling contrast analysis of a low angle sub-grain boundary, Scripta Mater. 109 (2015) 76-79.

[23] H. Mansour, M.A. Crimp, N. Gey, X. Iltis, N. Maloufi, Dislocation Analysis of a Complex Sub-grain Boundary Using Accurate Electron Channelling Contrast Imaging in a Scanning Electron Microscope, in press.

[24] L. Germain, D. Kratsch, M. Salib, N. Gey, Identification of sub-grains and low angle boundaries beyond the angular resolution of EBSD maps, Mater. Char. 98 (2014) 66-72.

[25] B. Beausir, J.-J. Fundenberger, Université de Lorraine - metz, ATEX® - analysis tools for electron and X-ray diffraction. http://atex-software.eu/, 2015.

[26] H. Mansour, J. Guyon, M.A. Crimp, N. Gey, B. Beausir, N. Maloufi, Accurate electron contrast analysis of dislocations in fine grained bulk materials, Scripta Mater. 84-85 (2014) 11-14.

[27] J. Guyon, H. Mansour, N. Gey, M.A. Crimp, S. Chalal, N. Maloufi, Sub-micron resolution selected area electron channeling patterns, Ultramicroscopy 149 (2015) 34-44.

[28] L. Portelette, B. Michel, M. Ibrahim, E. Castelier, M. Ben Saada, X. Iltis, P. Garcia, Numerical Simulation of the $\mathrm{UO}_{2}$ Viscoplasticity at the Polycristal Scale : Microscopic Validation, vol 16, EMMC, Nantes, France, 2018. March 26-28.

[29] P. Garcia, A. Miard, J.B. Parise, M. Ben Saada, X. Iltis, C. Introni, T. Helfer, High temperature creep of uranium dioxide: on the influence of equilibrium oxygen partial pressure, MRS Spring Meet. (2018). April 2-6, Phoenix, USA.

[30] S. Demouchy, A. Mussi, F. Barou, A. Tommasi, P. Cordier, Viscoplasticity of polycrystalline olivine experimentally deformed at high pressure and $900^{\circ} \mathrm{C}$, Tectonophysics 623 (2014) 123-135.

[31] G.M. Pennock, M.R. Drury, Low angle subgrain misorientations in deformed $\mathrm{NaCl}$, J. Microsc. 217 (2005) 130-137.

[32] G.M. Pennock, M.R. Drury, C.J. Spiers, The development of subgrain misorientations with strain in dry synthetic $\mathrm{NaCl}$ measured using EBSD, J. Struct. Geol. 27 (2005) 2159-2170.

[33] M.P. Staiger, I. Kolbeinsson, J. Newman, T. Woodfield, T. Sato, Orientation imaging microscopy of polycrystalline sodium chloride, Mater. Char. 61 (2010) 413-419.

[34] S.L.A. Valcke, G.M. Pennock, M.R. Drury, J.H.P. De Bresser, Electron backscattered diffraction as a tool to quantify subgrains in deformed calcite, J. Microsc. 224 (2006) 264-276. 